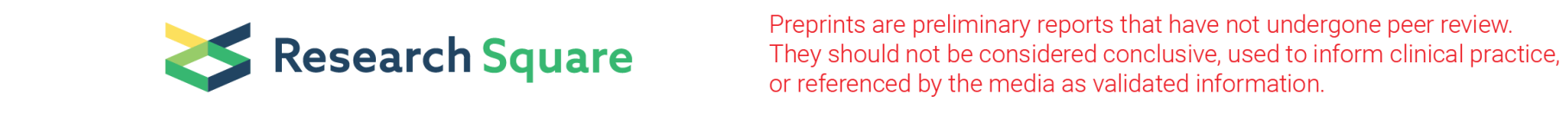

\title{
Effectiveness of dietetic intervention for people with type 2 diabetes: a meta-analysis
}

George Siopis ( $\nabla$ gsio7745@uni.sydney.edu.au )

The University of Sydney https://orcid.org/0000-0003-1407-0846

\section{Stephen Colagiuri AO}

University of Sydney

Margaret Allman-Farinelli

University of Sydney

\section{Original investigation}

Keywords: Clinical effectiveness, Diet, Dietitian, Dietetic counselling, Medical nutrition therapy, Meta-analysis, Metabolic syndrome, Nutrition intervention, Systematic review, Type 2 diabetes mellitus

Posted Date: September 14th, 2020

DOI: https://doi.org/10.21203/rs.3.rs-59641/v1

License: () (1) This work is licensed under a Creative Commons Attribution 4.0 International License. Read Full License

Version of Record: A version of this preprint was published at Clinical Nutrition on May 1st, 2021. See the published version at https://doi.org/10.1016/j.clnu.2020.12.009. 


\section{Abstract \\ Background}

Diet is central to treatment of type 2 diabetes. This review aimed to compare the effectiveness of nutrition therapy delivered by dietitians to nutrition advice delivered by other healthcare professionals in adults with type 2 diabetes on metabolic parameters.

\section{Methods}

Cochrane CENTRAL, CINAHL, EMBASE, MEDLINE and PsychINFO were searched for randomised controlled trials of three months duration or longer, published from 1st January 2008 to 18th June 2019. Relevant data were extracted from studies with additional author information. Random-effects meta-analysis assessed mean changes in HbA1c and other clinical parameters. PROSPERO registration number: CRD42019130528.

\section{Results}

Of 2477 records identified, fourteen studies $(n=3338)$ were eligible for qualitative synthesis and meta-analysis. The mean changes [95\% $\mathrm{Cl}]$ at follow-up in HbA1c, BMI, weight, LDL cholesterol, systolic and diastolic blood pressure were $-0.47[-0.92,-0 \cdot 02] \%,-0.38[-0 \cdot 63$, $-0 \cdot 13] \mathrm{kg} / \mathrm{m}^{2},-1 \cdot 49[-2 \cdot 14,-0 \cdot 84] \mathrm{kg},-0 \cdot 15[-0 \cdot 33,0.04] \mathrm{mmol} / \mathrm{L},-0 \cdot 75[-2 \cdot 45,0.96] \mathrm{mm} \mathrm{Hg}$ and $-1 \cdot 17[-4 \cdot 52,2 \cdot 17] \mathrm{mm}$ Hg respectively in favour of the intervention group.

\section{Conclusions}

Nutrition therapy provided by dietitians was associated with better clinical parameters of type 2 diabetes, including clinically significant improved glycaemic control, across diverse multiethnic patient groups from all six inhabited continents. This conclusion should be reflected in clinical guidelines.

\section{Background}

Type 2 diabetes mellitus has emerged as a global epidemic, affecting around half a billion people, with an estimated cost in excess of $\$ 1 \cdot 3$ trillion. ${ }^{1-3}$ Nutrition therapy is central for the treatment of type 2 diabetes, due to its beneficial effects on regulating glucose concentrations and other metabolic parameters, as well as facilitating weight control in synergy with physical activity. 4,5

International guidelines emphasise the importance of medical nutrition therapy, delivered by accredited dietitians, for people with type 2 diabetes. ${ }^{6-8}$ However, individuals with type 2 diabetes may receive their nutritional advice from other healthcare professionals, for example physicians, ${ }^{9-11}$ despite global reports highlighting a lack of nutrition education in medical curricula. ${ }^{12,13}$ There is some evidence that dietetic intervention led by accredited dietitians achieves better clinical parameters than nutritional advice provided by a doctor or nurse. ${ }^{14}$

This review is the first to include data from all six inhabited continents to expand our knowledge on the effect of dietetic counselling provided to individuals with type 2 diabetes by an accredited dietitian, compared to nutrition advice provided by other healthcare professionals. We conducted a systematic literature review for randomised controlled trials (RCTs) and a meta-analysis to quantify the different effects on haemoglobin A1c (HbA1c), body mass index (BMI), weight, low-density lipoprotein (LDL) cholesterol and blood pressure (BP).

\section{Methods}

\section{Search strategy and selection criteria}

The protocol and reporting of this systematic review and meta-analysis were consistent with the PRISMA guidelines. ${ }^{15}$ Cochrane CENTRAL, CINAHL, EMBASE, MEDLINE and PsychINFO were searched using broad search terms, including combinations of 'diabetes', 'diabetes mellitus', 'type 2 diabetes', 'type 2 diabetes mellitus', 't2d', 't2dm', 'tiid', 'tiidm', 'diet', 'nutrition', 'intervention', 'counselling', 'advice', 
'therapy' and 'obese'. The complete search strategy used for the databases is presented in the appendix ( $p$ 2). The reference list of relevant studies retrieved through database searches was also hand searched to identify additional studies. Grey literature and trial registries were searched and experts were contacted for unpublished data. The systematic review methodology was registered in PROSPERO (CRD42019130528). The protocol is available at https://www.crd.york.ac.uk/PROSPERO/display_record.php? RecordID=130528.

Citations and abstracts of all retrieved studies were downloaded to ENDNOTE X8 citation management software (Clarivate Analytics, PA, USA). Duplicates were removed and the remaining studies were assessed for eligibility criteria by two researchers (GS and MAF). Randomised controlled trials (RCTs), pseudo-RCTs and cluster RCTs of type 2 diabetes management programmes in adults, implementing medical nutrition therapy delivered by a dietitian and published in the English language during the period from 1 st January 2008 to 18th June 2019 were considered for inclusion. To reflect current practice, contemporary publications after the year of 2008 were considered, as this was the year that the Academy of Nutrition and Dietetics evidence-based nutrition practice guidelines were updated. ${ }^{16}$ A more recent version has been released, ${ }^{17}$ but the timeframe of two years since 2017 does not allow for a substantial body of studies to be examined

\section{Data analysis}

A data extraction form was developed based on the guidelines in the 6th version of the Cochrane Handbook for Systematic Reviews of Interventions. ${ }^{18}$ The form was piloted on all the included studies and refined before being used to extract the data: authors' details (names, affiliations, funding), study details (year and country, design, purpose, inclusion criteria, blinding and randomisation method, statistics, validity of outcome measures, conclusions made considering study limitations), participants' characteristics (sample size, recruitment process, retention rate, compliance, demographics), intervention (primary and secondary outcome factors, duration).

The primary endpoint was 3 -months or more change in the mean $[95 \% \mathrm{Cl}$ ] of the plasma concentration of haemoglobin $\mathrm{A} 1 \mathrm{c}(\mathrm{HbA} 1 \mathrm{C})$. Secondary endpoints of interest included lipid profile, blood pressure (BP), fasting glucose levels, insulin concentration, decreases in medication, as well as anthropometric outcomes (BMl, weight and waist circumference) and changes in nutrients and food groups.

The Cochrane risk-of-bias (ROB) tool was used to assess the quality of the studies on aspects of selection (random-sequence generation and allocation concealment), performance and detection (blinding of participants, personnel, and assessors), appropriateness of analysis (selective outcome reporting), and other sources of bias in accordance with the recommendations of the Cochrane Collaboration. Studies were ranked by two authors (GS and MAF) as "low risk", "high risk", or "unclear" (either a lack of information or uncertainty over a potential bias). ${ }^{18}$ Authors resolved discrepancies by discussion.

Within groups difference in means for HbA1c, BMl, weight, LDL cholesterol, systolic and diastolic BP and their standard deviations (SD) for intervention and control groups were entered into Review Manager (RevMan) v5.3 software (Copenhagen: The Nordic Cochrane Centre, The Cochrane Collaboration, 2014). To increase the precision of the point estimate, when only between group and not within group differences were reported, the latter were requested from the authors. If standard error (SE) was reported instead of SD, then this was converted to $S D$ using the formula: $S D=S E * \sqrt{n}$. If neither $S D$ nor $S E$ could be obtained from the published data, or following communication with the authors, then the SD was imputed according to Cochrane's recommendations ${ }^{19}$ (appendix $p$ 10). The effect sizes and SEs of the studies were pooled using the random-effects model. The random-effects meta-analysis model was selected, despite resulting in wider confidence intervals around point estimates, since heterogeneity is expected due to the differences in study populations and procedures. ${ }^{20}$

The assumption of homogeneity of true effect sizes was assessed by the Cochran $Q$ test ${ }^{21}$ and the degree of inconsistency across studies $(R)$ was calculated. ${ }^{22} R$ describes the percentage of total variation across studies that is due to heterogeneity rather than sampling error and ranges between $0 \%$ (no inconsistency) and 100\% (high heterogeneity) with values between $0-40 \%, 30-60 \%, 50-90 \%$ and $75-100 \%$ suggesting low, moderate, substantial and considerable heterogeneity. ${ }^{18,22}$

Publication bias was assessed informally by visually inspecting a funnel plot of the mean change in HbA1c, BMI, weight, LDL cholesterol, or BP plotted against the corresponding standard error, on the assumption that interventions achieving HbA1c, BMI, weight, LDL cholesterol or BP reductions and with larger samples were more likely to be published. ${ }^{18}$

The quality of evidence for each outcome was assessed using Grading of Recommendations, Assessment, Development and Evaluation (GRADE) criteria $^{23}$ (appendix $\mathrm{p} 11$ ). 


\section{Results}

The search yielded 2477 records. Two records were identified through hand-searching the reference lists. Six hundred and nineteen duplicates (619) were removed and the remaining 1860 abstracts were assessed for eligibility (Fig. 1). One thousand seven hundred and ninety four (1794) abstracts were excluded during the primary exclusion round and a further 52 were excluded after assessing the full text articles. The reasons for exclusion of the full articles are shown in the appendix ( $p$ 12). Fourteen studies ${ }^{24-37}$ were included in the qualitative synthesis and thirteen of them in the meta-analysis. ${ }^{24-31,33-37}$ Maindal et al. ${ }^{32}$ was not possible to be included in the metaanalysis due to missing values. The author responded to the initial inquiry to provide the requested data but did not follow through. Two reminder email requests were sent to no avail. Three of these studies $24,26,28$ have been cited in previous nutrition guidelines for diabetes management. ${ }^{38}$

All but one $e^{29}$ trial were conducted in outpatient settings. Thirteen trials involved a parallel RCT design ${ }^{24-29,31-37}$ and one trial ${ }^{30}$ cluster randomization with each cluster representing a community. In total, 3338 subjects with type 2 diabetes, representing a diverse range of participants from eighteen countries from the six inhabited continents, were included (appendix p 20 and 27). Only the "diet" group that was randomly assigned to nutrition therapy $(n=248)$, and not the "diet plus activity" group $(n=246)$, was included from the study by Andrews et al. ${ }^{24}$

Table 1 summarizes the study characteristics. Supplementary tables 1, 2 and 3 (appendix pp 22-30) list details of each study and a summary of participants' demographics and clinical characteristics. The mean age of participants was $55 \cdot 8$ years. The majority of participants were of white European ethnic background (40\%), with one quarter Asian and $12 \%$ black African. Approximately half of the participants were males (49\%) and half females (51\%). One-third of the participants (35\%) had completed less than high-school education and half (53\%) were employed. Participants were on average diagnosed with type 2 diabetes for six years and 10 weeks and about one in ten was a self-reported smoker. The mean BMI at baseline was $31 \cdot 5 \mathrm{~kg} / \mathrm{m}^{2}$ and the mean HbA1c was $7 \cdot 89 \%$. The majority of the participants were on oral hypoglycaemic agents (59\%), antihypertensives (68\%) and lipid modifying medication (51\%). No significant differences at baseline between the pooled intervention versus the control group were observed.

For the individual studies, there were few significant differences in baseline characteristics between groups. In the study by Huang et al., ${ }^{28}$ patients in the control group had higher BMI and diastolic BP than those of the intervention group at baseline. In two studies, ${ }^{31,37}$ the intervention group had at least twice as many smokers as the control group. In the study by Eakin et al. ${ }^{27}$ the control group was of a lower education status. Baseline BMI was much lower in the four Asian studies ${ }^{28,30,36,37}$ than in the eight studies of non-Asians. ${ }^{24-27}$, 29,31-35 $\mathrm{HbA} 1 \mathrm{c}$ was just above normal at baseline in one study, ${ }^{32}$ close to normal in another one, ${ }^{24}$ just $<8 \%$ in five studies, ${ }^{27,30,31,34,35}$ and $>8 \%$ in the other seven studies. ${ }^{25,26,28,29,33,36,37}$

Interventions lasted from four months to three years. In most studies (79\%), the participants consulted with the dietitians at least six times. A few studies reported on the duration of each session, but in most this was not clear. Sessions were either face-to-face, telephone, individual, group or a combination of some of the aforementioned. The reported dropout rate varied considerably, from $2 \%$ in the study by Andrews et al., ${ }^{24}$ to $20 \%$ in the study by Huang et al., ${ }^{28}$ but with no significant difference between groups. Shahid et al. ${ }^{36}$ did not report attrition.

Risk of bias was assessed at the study level using the Cochrane Risk of Bias tool. ${ }^{18}$ In all fourteen studies, subjects were randomly assigned before the intervention. The random-sequence-generation method was stated in all the studies but two. ${ }^{30,36}$ In the study by Liu et al., ${ }^{30}$ two Chinese communities were randomly assigned to either the intervention or a control. This assignment involves high risks of recruitment and selection bias. The study by Shahid et al. ${ }^{36}$ reported that patients were "randomly distributed" but there was no reference to a methodology. Allocation was not concealed, or it was unclear, in five studies. ${ }^{28-30,33,36}$ In one study doctors were unaware of allocation of participants but dietitians and nurses were. ${ }^{24}$ The blinding of participants and personnel (performance bias) was not possible in the present studies. The blinding of outcome assessors was reported in four studies. ${ }^{24,27,31,35}$ Handling of missing endpoints (e.g. intention-to-treat, multiple imputation) was not clear in some studies. ${ }^{28,29,30,37}$ The reporting in two trials was not consistent with their registration information $28,34,37$ and two trials were not registered. ${ }^{30,33}$ Three studies did not report conflict of interest. ${ }^{31,35,36}$ In one study that reported industry funding, authors stated affiliations with the funding body. ${ }^{34}$ No other potential sources of bias were identified from the studies (Fig. 2). Conflicts of interest and funding sources are listed in the appendix ( $\mathrm{p} 31$ ). The risk of bias ratings meant the overall rating of the body of evidence was downgraded despite all being RCTs. 
All trials reported changes in $\mathrm{HbA} 1 \mathrm{c}$, with nine reporting it as the primary outcome and three $27,32,34$ as a secondary outcome. Ten-year modelled cardiovascular risk was the primary outcome for Maindal et al. ${ }^{27}$ and changes in body weight for the other two. ${ }^{32,34}$ Ten studies reported changes in $\mathrm{BMI}$ and weight, twelve reported on blood pressure and eight on low-density lipoprotein (LDL) cholesterol. Less than half of the studies reported changes in energy and nutrient intake, medication use, waist circumference and physical activity. We pooled results for HbA1c, BMI, weight, LDL cholesterol and systolic and diastolic blood pressure (BP) in meta-analyses.

Table 2 shows the summary of findings of the meta-analysis. The GRADE scoring methodology is explained in the appendix ( $p$ 11). Figure 2 and supplementary Figs. 2-6 (appendix pp 32-36) show Forest plots of the primary and secondary outcomes respectively. Nutrition intervention delivered by dietitians achieved a $-0 \cdot 47[-0 \cdot 92,-0.02]$ percentage point greater reduction in $\mathrm{HbA} 1 \mathrm{c},-0 \cdot 38[-0 \cdot 63,-0 \cdot 13]$ $\mathrm{kg} / \mathrm{m}^{2}$ lower BMl, $-1 \cdot 49[-2 \cdot 14,-0 \cdot 84] \mathrm{kg}$ greater reduction in weight, $-0 \cdot 15[-0 \cdot 33,0.04] \mathrm{mmol} / \mathrm{L}$ lower LDL cholesterol and $-0.75[-2 \cdot 45$, $0 \cdot 96] \mathrm{mm} \mathrm{Hg}$ and $-1 \cdot 17[-4 \cdot 52,2 \cdot 17] \mathrm{mm} \mathrm{Hg}$ greater reductions in systolic and diastolic BP respectively, compared to nutrition advice by other healthcare professionals. The reductions in $\mathrm{HbA} 1 \mathrm{c}, \mathrm{BMI}$ and weight were statistically significant $(\mathrm{P}=0.04, \mathrm{P}=0.003$ and $\mathrm{P}<$ 0.00001 respectively). Heterogeneity between the studies was statistically significant $(Q=985 \cdot 48, P<0.00001)$ and "considerable" in magnitude $(R=99 \%)$ for the HbA1c, BMI $(\mathrm{Q}=53 \cdot 75, \mathrm{P}<0 \cdot 00001, R=85 \%)$, weight $(\mathrm{Q}=45 \cdot 44, \mathrm{P}<0.00001, R=80 \%), \mathrm{LDL}$ cholesterol $(\mathrm{Q}=$ 206.28, $\mathrm{P}<0.00001, P=97 \%)$, systolic $\mathrm{BP}(\mathrm{Q}=76 \cdot 45, \mathrm{P}<0.00001, P=87 \%)$ and diastolic $\mathrm{BP}(\mathrm{Q}=1686 \cdot 47, \mathrm{P}<0.00001, P=99 \%)$ outcomes. The body of evidence was considered inconsistent due to the "considerable" heterogeneity and the level of evidence was downgraded for all outcomes. A further point was deducted from the evidence score due to indirectness, as some studies had short duration and all of them reported surrogate endpoints.

Pooled participants in the intervention group $(n=1334)$ achieved more than four times the reduction in HbA1c, i.e. $-0.65 \%( \pm 0 \cdot 42)$, versus $-0.15 \%( \pm 0.39)$ that was achieved by the participants in the control group $(n=1177)$. In addition, pooled participants in the intervention group $(n=998)$ reduced their weight on average by about 1.35 kilogram $( \pm 0.75 \mathrm{~kg})$, whereas participants in the control group $(n=830)$ gained on average 78 grams ( \pm 910 grams) (data not shown). This weight reduction in the intervention group corresponded to approximately $2 \%$ of their baseline weight.

We conducted a series of secondary analyses excluding one of all possible combinations of two of, three of, four of, five of, six of and all seven of studies that scored poorly in the ROB assessment. ${ }^{28-30,33,34,36,37}$ None of the different combinations changed the direction of associations, but some of them affected the statistical significance of the HbA1c, BMI and weight outcomes (data not shown). When only the six studies $24-27,31,35$ that scored higher on ROB (at least five "low risk" fields in total and mandatory "low risk" assessment for randomisation process, detection bias and selective reporting) were analysed, the results remained statistically significant albeit attenuated, e.g. HbA1c was $-0 \cdot 29[-0 \cdot 50,-0 \cdot 07] \%$.

We performed another series of secondary analyses, this time including only the eight studies that followed up for at least 12 months. ${ }^{24,25,27,28,30,33-35}$ Removing the five datasets of the studies that followed up at six months or sooner ${ }^{26,29,31,36,37}$ attenuated the result for HbA1c $(-0.24[-0.41,-0.07] \%)$ but increased it for weight $(-1.62[-2.73,-0.51] \mathrm{kg})$. None of these changes were statistically significant and the point estimate gained precision. The directions of association and measured effects were not statistically different for the LDL cholesterol and blood pressure outcomes. On the contrary, the BMI outcome crossed the line of no-effect (data not shown).

The robustness of the estimate was examined by sequentially removing each study and reanalysing the remaining datasets. The estimated effect sizes ranged from -0.51 to $-0.30 \%$ for $\mathrm{HbA} 1 \mathrm{c} ;-0.47$ to $-0.30 \mathrm{~kg} / \mathrm{m}^{2}$ for BMl; -1.68 to $-1.13 \mathrm{~kg}$ for weight; -0.19 to $-0.07 \mathrm{mmol} / \mathrm{L}$ for LDL cholesterol; -1.50 to $-0.34 \mathrm{~mm} \mathrm{Hg}$ for systolic blood pressure; and $-1.51 \mathrm{for}-0.19 \mathrm{~mm} \mathrm{Hg}$ for the diastolic blood pressure outcome. Individual removal of any of the following datasets from Coppell et al., Liu et al., Lynch et al., or from Suriyawongpaisal et al. abolished the statistical significance of the result for the HbA1c. Removal of the Shahid et al. dataset rendered the LDL cholesterol result statistically significant. No single study had an impact on the significance of the effect size for the BMI, weight, systolic and diastolic blood pressure outcomes (data not shown).

We also tested whether the fixed-effects model would produce different results to the random-effects one. The fixed-effects model rendered the LDL cholesterol and blood pressure outcomes statistically significant and maintained statistical significance in the HbA1c, weight and BMI outcomes, augmenting the effect size of HbA1c. It resulted in narrower confidence intervals, as expected.

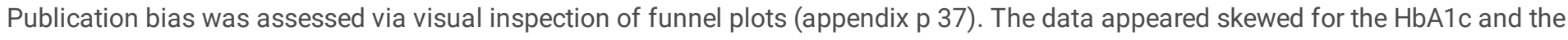
diastolic BP outcomes. The asymmetry observed for the HbA1c is likely a result of the heterogeneity. ${ }^{39}$ Whereas the skewed distributions seen for the diastolic BP outcome is largely due to an excess of medium to large studies with negative effects on the outcome. The 
asymmetry manifested in the weight outcome is likely due to an excess of small to medium studies with positive effects on the outcome. There was no evidence of publication bias for systolic BP. Publication bias could not be assessed for the BMI and LDL cholesterol outcomes as the number of studies is not adequate to distinguish chance from real asymmetry. ${ }^{19}$

\section{Discussion}

Type 2 diabetes is a major growing global health challenge. Medical nutrition therapy (MNT) has been shown to improve clinical parameters of type 2 diabetes. Our aim was to compare MNT delivered by accredited dietitians to nutrition advice delivered by other healthcare professionals (verbal or written). In this systematic review, we identified fourteen RCTs from eighteen countries from all six inhabited continents, involving 3338 participants with diverse backgrounds, that compared nutrition interventions for people with type 2 diabetes delivered by accredited dietitians with nutrition advice delivered by other healthcare professionals. The meta-analysis of thirteen studies found improved outcomes when nutrition intervention was delivered by a dietitian compared with nutrition advice delivered by other healthcare professionals. Dietitian-delivered MNT achieved a statistically as well as clinically significant reduction in $\mathrm{HbA1c}$, with participants in the intervention group reducing their $\mathrm{HbA} 1 \mathrm{c}$ four-and-a-half times more than participants in the control group. In addition, nutrition therapy achieved statistically significant reductions in BMI and weight in favour of the dietitian-led intervention. However, the overall magnitudes of change in BMI and weight were small and neither was considered clinically significant, but the estimate was precise as reflected by the narrow confidence intervals. Substantial heterogeneity was observed for all outcomes, as expected when combining data from eighteen countries with different health systems, guidelines and practices. In addition to the heterogeneity, the indirectness of reporting surrogate outcomes and the publication and risk of bias, resulted in the overall strength of evidence being rated very low.

Nutrition intervention delivered by dietitians achieved greater reductions for all outcomes, compared with when nutrition advice was delivered by other health care professionals in written or verbal form, with the reductions for $\mathrm{HbA1c}$, BMI and weight being statistically significant and the LDL cholesterol narrowly missing this classification. However, only the reduction for $\mathrm{HbA} 1 \mathrm{c}$ was clinically significant. ${ }^{40-42}$ A reduction in $\mathrm{HbA} 1 \mathrm{c}$ of half a percentage point $(0.5 \%)$ is considered clinically significant, ${ }^{40,41}$ which is consistent with the $0.47 \%$ reduction in $\mathrm{HbA} 1 \mathrm{c}$ in this review. A $3-5 \%$ of reduction in body weight is considered clinically significant, ${ }^{42,43}$ but this was not demonstrated in this analysis. It has been previously argued that even small reductions in the concentrations of metabolic syndrome parameters can yield clinically significant improvements due to their synergistic effects. ${ }^{44}$ Therefore, although not attaining the conventional clinically significant cut-off point, the weight reduction seen in the intervention group may be clinically meaningful, especially when considering that the intervention group achieved a greater reduction than the control group. Factoring in the observations that participants in the intervention group also reduced all other parameters, i.e. LDL cholesterol and blood pressure compared with the control group, and achieved a clinically significant reduction in $\mathrm{HbA} 1 \mathrm{c}$, the synergistic interplay between these metabolic parameters may be clinically meaningful. Our analysis indicated that participants in the intervention group reduced their weight by about $2 \%$, which is close to the suggested minimum reduction of $2.5 \%$ that was shown to produce improvement in glycaemic outcomes in the Look AHEAD trial. ${ }^{45}$ Future, longitudinal studies in people with type 2 diabetes should further explore the effects of multi-metabolic-parameter improvements in the overall manifestation of diabetes related co-morbidities and complications.

Sensitivity analyses showed no effect on the direction of associations for the HbA1c outcome, when poorly ROB-scoring studies were excluded, or when only long-term (one year or more) studies were included. However, the magnitude of the change in effect size was attenuated and was no longer clinically significant. The heterogeneity was considerable, indicating that the weight that even large trials receive is reduced in the pooled analysis and thus even adding another large trial in the future will only have a small effect on the pooled result and is unlikely to alter the conclusions of the present meta-analysis.

The current findings are consistent with the only other previous meta-analysis that compared nutrition intervention delivered by a dietitian to nutrition advice delivered by other health care professionals. ${ }^{14}$ In this review, we extend the evidence presented by Moller et al. Data from 12 more countries, i.e. Argentina, Australia, Germany, India, Poland, Serbia, Slovakia, Slovenia, South Africa, Spain, Thailand and Turkey, as well as from four more sites in the USA, are included to further inform our understanding of the effectiveness of MNT on clinical parameters of type 2 diabetes, in a variety of geographic locations, economies, health care systems, as well as cultures considering the interwoven nature of culture and diet. We have focused on interpreting the data's clinical relevance, with the objective to inform and improve clinical practice. Our findings further support the international guidelines that recommend involvement of the dietitian in the management of diabetes. ${ }^{6-8}$ Physicians have previously reported a lack of education in medical curricula throughout the world as well as a lack of confidence in providing nutritional counselling to patients. ${ }^{12,13,46}$ Dietitians receive extensive nutrition 
education and are therefore better informed to recommend optimal diets for the current ${ }^{47,48}$ and future ${ }^{49}$ management of type 2 diabetes. As the International Diabetes Federation guidelines state, "referral to a dietitian can be very useful to learn how to translate the caloric restriction into feasible meals and how to manage some barriers for caloric restriction such as emptiness and hunger". ${ }^{8}$ A 2017 review of nutrition interventions by dietitians showed effectiveness in glycaemic control and weight loss but not in correcting atherogenic dyslipidemia. ${ }^{50}$ This agrees with our findings, as well as those of others, ${ }^{51}$ that did not show any significant improvement in LDL cholesterol concentration.

It will be useful to determine the optimal number of visits to the dietitian required to elicit meaningful results. In our studies, most participants saw the dietitians about six to eight times. It has previously been reported that Australian dietitians believe that people with type 2 diabetes need to consult with the dietitian at least four times per annum. ${ }^{52} \mathrm{In}$ addition to the frequency of visits, the average duration of the visit required to achieve optimal metabolic results should be determined. Few of the studies included in our meta-analysis reported the duration of each session, and in most studies this was not clear. Dietetic consult session durations vary around the world with reports indicating sessions of an average of 45-60 minutes in Australia, ${ }^{53}$ fifty minutes in Canada ${ }^{54}$ and about half an hour in Israel. ${ }^{55}$ Patients appear to prefer longer sessions. ${ }^{56}$ Of interest, government guidelines advise a minimum of twenty minutes per session in Australia ${ }^{57}$ and about 30-40 minutes in the UK. ${ }^{58}$ It will also be useful to examine the frequency of visits and costeffectiveness of treatments. Previous reports have demonstrated returns-on-investment in the order of about NZ\$5.50-\$99 for every NZ\$1 spent on dietetic intervention in New Zealand ${ }^{59}$ and $€ 14$ to $€ 63$ for every $€ 1$ in the Netherlands. ${ }^{6}$ It will be interesting to assess whether the cost effectiveness can be further improved by optimising dietetic consult time and frequency, as well as to investigate other factors that may mediate and/or moderate the cost effectiveness of services, such as further use of telehealth, especially in areas with poor access to services. ${ }^{61}$

The strengths of the current analyses lie in the use of standard methodology as documented in the PRISMA and Cochrane guidelines for conducting systematic reviews and meta-analyses; the fact that a demographically and culturally diverse cohort from eighteen countries from all six inhabited continents was included; the inclusion of additional data from lower and upper middle income countries; and the comprehensive series of sensitivity analyses performed to reinforce the main findings. The narrow confidence intervals underline the great precision of the effect estimates. Møller et al. combined within-group differences in means with those of between-group ones in their meta-analyses, as a mixture of these were available in the published articles. ${ }^{14}$ We communicated with the authors to consult with them on unpublished data and accurately calculate within-group differences in means for the meta-analysis, to increase the precision of the point estimate.

Our analyses also have limitations. We searched five databases for studies in adults. The adult filter for Medline is $>19$ years of age, whereas for EMBASE and PsychINFO it is > 18 years old. This means that articles listed in MEDLINE with participants between 1819 years old may have been excluded. It is crucial to ensure consistency between the different databases in terms of how an "adult" person is defined to avoid such discrepancies. We also restricted the search to articles in English-language. It has been previously reported that the proportion of RCTs with positive results published in English-language journals is almost twice those published in German-language journals. ${ }^{62}$ Therefore, this may present a source of bias due to the potential overestimation of the reported intervention effect. However, other reports indicate a difference in just one out of 36 meta-analyses when the non-English publications are included. ${ }^{63}$ Our publication bias analysis indicated asymmetrical distributions for three outcomes. Some of the included studies presented methodological limitations, for example the cluster randomisation protocol. Despite this, there was no evidence of reporting substantially different effects. All of the outcomes presented substantial heterogeneity. It has been proposed that pooling data from multiple heterogeneous trials and presenting a single summary estimate can be misleading. ${ }^{64}$ However, we undertook several sensitivity analyses to test the robustness of the outcomes and the influence of individual and combinations of studies on these with no significant changes in our inferences. Statisticians also argue that since clinical and methodological diversity always occur in a meta-analysis, statistical heterogeneity is inevitable. ${ }^{19}$ Finally, the random effects model has been criticized for its limitation in taking covariates into account to explain heterogeneity. ${ }^{65}$ However, carrying out the analysis using the fixed-effects model did not produce any statistically significant different results for the $\mathrm{HbA1c}, \mathrm{BMI}$ and weight outcomes. We therefore conclude that our methodology is robust and sound.

\section{Conclusions}

In summary, dietitian-delivered nutrition therapy for people with type 2 diabetes achieves better outcomes than nutrition advice delivered by other healthcare professionals. Improvements in glycaemic control and body weight are statistically significant and the former clinically significant as well. Future longitudinal studies in people with type 2 diabetes should further explore the effects of multi- 
metabolic-parameter improvements in the overall phenotypic manifestation of co-morbidities to elucidate the long-term effects of the interventions and inform clinical practice. The optimal length and frequency of dietetic consultations that produces clinically meaningful results in the most cost-efficient manner merits further investigation.

\section{Abbreviations}

$95 \% \mathrm{Cl}, 95 \%$ confidence interval

BMI, body mass index

BP, blood pressure

GRADE, Grading of Recommendations, Assessment, Development and Evaluation

HbA1c, haemoglobin A1c

$I^{2}$, inconsistency measure

LDL, low-density lipoprotein

PRISMA, preferred reporting items for systematic reviews and meta-anlyses

$\mathrm{RCT}$, randomised controlled trials

$\mathrm{ROB}$, risk of bias

SD, standard deviation

SE, standard error

\section{Declarations}

\section{Ethics approval and consent to participate}

Not applicable.

\section{Consent for publication}

All authors have read the manuscript and provide consent for publication.

\section{Availability of data and materials}

The datasets used and analysed during the current study are available from the corresponding author on reasonable request.

\section{Competing interests}

The authors declare that they have no competing interests.

\section{Funding}

This research received no specific grant from any finding agency in the public, commercial or not-for-profit sectors.

\section{Authors' contributions}

All authors conceived the idea for the review and designed the review protocol. GS and MAF searched the literature and extracted the data. GS analysed the data. All authors interpreted the data. GS drafted the manuscript. All authors critically reviewed and revised the manuscript. 


\section{Acknowledgements}

The authors would like to thank University of Sydney's academic liaison librarian for advising with the literature search strategy and assisting with the database searches. We also thank the authors, statisticians and custodians of the data of the reviewed studies that kindly provided further data for the meta-analysis, namely Dr Clare England and Dr Sam Leary (Andrews et al.), Mr Marc Vacquier (Benson et al.), Prof Kirsten Coppell (Coppell et al.), Prof Elizabeth Eakin and Dr Elisabeth Winkler (Eakin et al.), Prof Elizabeth Lynch and Ms Elizabeth Avery (Lynch et al.), and Dr Thomas Keyserling (Samuel-Hodge et al.) as well as those that preferred to remain anonymous. This research did not receive any specific grant from funding agencies in the public, commercial, or not-for-profit sectors. George Siopis is a recipient of the University of Sydney International Strategic (USydIS) fund scholarship and of the Neville Whiffen scholarship. Neither of these bodies had any role in the design and conduct of the study; collection, management, analysis, and interpretation of the data; preparation, review, or approval of the manuscript; or decision to submit the manuscript for publication.

\section{References}

1. World Health Organisation. Diabetes fact sheet (2018). Available from https://www.who.int/news-room/fact-sheets/detail/diabetes (accessed on September 5, 2020).

2. International Diabetes Federation. IDF Diabetes Atlas $9^{\text {th }}$ edition (2019). Available from https://www.diabetesatlas.org/en/sections/worldwide-toll-of-diabetes.html (accessed on September 5, 2020).

3. Bommer C, Heesemann E, Sagalova V, Manne-Goehler J, Atun R, Bärnighausen T, Vollmer S. The global economic burden of diabetes in adults aged 20-79 years: a cost-of-illness study. Lancet Diabetes Endocrinol. 2017; 5: 423-430.

4. Lean ME, Leslie WS, Barnes AC et al. Primary care-led weight management for remission of type 2 diabetes (DiRECT): an open-label, cluster-randomised trial. Lancet 2017; 391: 541-551.

5. Delahanty L, Dalton KM, Porneala B et al. Improving diabetes outcomes through lifestyle change-A randomized controlled trial. Obesity 2015; 23: 1792-1799.

6. American Diabetes Association. 5. Lifestyle management. Standards of Medical Care in Diabetes. Diabetes Care2019; 42: S1: S46S60.

7. Diabetes UK. Evidence-based nutrition guidelines for the prevention and management of diabetes (2018). Available from https://diabetes-resources-production.s3.eu-west-1.amazonaws.com/resources-s3/2018-03/1373_Nutrition\%20guidelines_0.pdf (accessed on September 5, 2020).

8. International Diabetes Federation. IDF Clinical Practice Recommendations for managing Type 2 Diabetes in Primary Care (2017). Available from file:///C:/Users/gsio7745/Downloads/T2D-primary-care-guideline-print\%20(1).pdf (accessed on September 5, 2020).

9. Ball L, Desbrow B, Leveritt M. An exploration of individuals' preferences for nutrition care from Australian primary care health professionals. Aust J Prim Health2014; 20: 113-120.

10. Al Lenjawi B, Mohamed H, Amuna P, Zotor F, Ziki MD. A nurse-led theory-based educational intervention improves glycemic and metabolic parameters in South Asian patients with type II diabetes: a randomized controlled trial. Diabetology Internationa/ 2017; 8: 95-103.

11. Beyazit E, Mollaoglu M. Investigation of effect on glycosylated hemoglobin, blood pressure, and body mass index of diabetes intensive education program in patients with type 2 diabetes mellitus. Am J Men Health 2011; 5: 351-357.

12. Aggarwal M, Devries S, Freeman AM, et al. The Deficit of Nutrition Education of Physicians. Am J Med 2018; 131: 339-45.

13. Crowley J, Ball L, Hiddink GJ. Nutrition in medical education: a systematic review. Lancet Planet Health 2019; 3: e379-e389.

14. Møller G, Andersen HK, Snorgaard O. A systematic review and meta-analysis of nutritiontherapy compared with dietary advice in patients with type 2 diabetes. Am J Clin Nutr 2017; 106: 1394-1400.

15. Liberati A, Altman DG, Tetzlaff $\mathrm{J}$ et al. The PRISMA statement for reporting systematic reviews and meta-analyses of studies that evaluate healthcare interventions: explanation and elaboration. BMJ2009; 339: b2700.

16. Franz MJ, Boucher JL, Green-Pastors J, Powers MA. Evidence-based nutrition practice guidelines for diabetes and scope and standards of practice. J Am Diet Assoc 2008; 108. S1: S52-58.

17. MacLeod J, Franz MJ, Handu D, Gradwell E, Brown C, Evert A, Reppert A, Robinson M. Academy of Nutrition and Dietetics Nutrition Practice Guideline for Type 1 and Type 2 Diabetes in Adults: Nutrition Intervention Evidence Reviews and Recommendations. $J$ Acad Nutr Diet2017; 117: 1637-1658.

Page 9/13 
18. Higgins JPT, Thomas J, Chandler J, et al. Cochrane Handbook for Systematic Reviews of Interventionsversion 6.0 (updated July 2019). Cochrane, 2019. Available from training.cochrane.org/handbook (accessed on September 5, 2020).

19. Higgins JPT and Green S. Cochrane Handbook for Systematic Reviews of Interventionsversion 5.1 (updated March 2011 ). Cochrane, 2011. Available from https://handbook-5-1.cochrane.org/front_page.htm (accessed on September 5, 2020).

20. DerSimonian R, Laird N. Meta-analysis in clinical trials. Control Clin Trials 1986; 7: 177-188.

21. Cochran WG. The comparison of percentages in matched samples. Biometrika 1950; 37: 256-266.

22. Higgins JP, Thompson SG, Deeks JJ, Altman DG. Measuring inconsistency in meta-analyses. BMJ 2003; 327: 557-560.

23. Guyatt GH, Oxman AD, Sultan S, et al. GRADE guidelines. J Clin Epidemiol 2011; 64: 383-1316.

24. Andrews RC, Cooper AR, Montgomery AA, et al. Diet or diet plus physical activity versus usual care in patients with newly diagnosed type 2 diabetes: the early ACTID randomized controlled trial. Lancet 2011; 378: 129-139.

25. Benson GA, Sidebottom A, Hayes J, et al. Impact of ENHANCED (diEtitiaNs Helping pAtieNts CarEfor Diabetes) Telemedicine Randomized Controlled Trial on Diabetes Optimal Care Outcomes in Patients with Type 2 Diabetes. J Acad Nutr Diet 2019; 119: 585598.

26. Coppell KJ, Kataoka M, Williams SM, Chisholm AW, Vorgers SM, Mann JI. Nutritional intervention in patients with type 2 diabetes who are hyperglycaemic despite optimized drug treatment - Lifestyle Over and Above Drugs in Diabetes (LOADD) study: randomized controlled trial. BMJ 2010; 341: c3337.

27. Eakin EG, Winkler EA, Dunstan DW, et al. Living well with diabetes: 24-month outcomes from a randomized trial of telephonedelivered weight loss and physical activity intervention to improve glycemic control. Diabetes Care. 2014; 37: 2177-2185.

28. Huang MC, Hsu CC, Wang HS, Shin SJ. Prospective randomized controlled trial to evaluate effectiveness of registered dietitian-led diabetes management on glycemic and diet control in primary care setting in Taiwan. Diabetes Care 2010; 33: $233-239$.

29. Kattelmann KK, Conti K, Ren C. The medicine wheel nutrition intervention: a diabetes education study with the Cheyenne River Sioux Tribe. J Am Diet Assoc. 2009; 109: 1532-1539.

30. Liu H, Zhang M, Wu X, Wang C, Li Z. Effectiveness of a public dietitian-led diabetes nutrition intervention on glycemic control in a community setting in China. Asia Pac J Clin Nutr 2015; 24: 525-532.

31. Lynch EB, Liebman R, Ventrelle J, Avery EF, Richardson D. A self-management intervention for African Americans with comorbid diabetes and hypertension: a pilot randomized controlled trial. Prev Chronic Dis. 2014; 11: E90.

32. Maindal HT, Carlsen AH, Lauritzen T, Sandbaek A, Simmons RK. Effect of a participant-driven health education programme in primary care for people with hyperglycaemia detected by screening: 3-year results from the Ready to Act randomized controlled trial (nested within the ADDITION-Denmark study). Diabet Med. 2014; 31: 976-986.

33. Muchiri JW, Gericke GJ, Rheeder P. Effect of a nutrition education programme on clinical status and dietary behaviours of adults with type 2 diabetes in a resource-limited setting in South Africa: a randomised controlled trial. Public Health Nutr2016; 19: 142-155.

34. Niswender K, Piletic M, Andersen H, Conradsen Hiort L, Hollander P. Weight change upon once-daily initiation of insulin detemir with or without dietary intervention in overweight or obese insulin-naïve individuals with type 2 diabetes: results from the DIET trial. Diabetes Obes Metab. 2014; 16: 186-192.

35. Samuel-Hodge CD, Keyserling TC, Park S, Johnston LF, Gizlice Z, Bangdiwala SI. A randomized trial of a church-based diabetes selfmanagement program for African Americans with type 2 diabetes. Diabetes Educ. 2009; 35: 439-454.

36. Shahid M, Mahar SA, Shaikh S, Shaikh ZU. Mobile phone intervention to improve diabetes care in rural areas of Pakistan: a randomized controlled trial. J Coll Physicians Surg Pak. 2015; 25: 166-171.

37. Suriyawongpaisal P, Tansirisithikul R, Sakulpipat T, Charoensuk P, Aekplakorn W. A Participatory Randomized Controlled Trial in Knowledge Translation (KT) to Promote the Adoption of Self-Monitoring of Blood Glucose for Type 2 Diabetes Mellitus Patients in An Urban District of Thailand. J Med Assoc Thai. 2016; 99: 125-132.

38. Evert AB, Boucher JL, Cypress M, Dunbar SA, Franz MJ, Mayer-Davis EJ, Neumiller JJ, Nwankwo R, Verdi CL, Urbanski P. Nutrition therapy recommendations for the management of adults with diabetes. Diabetes Care 2013; 36: 3821-3842.

39. Terrin N, Schmid CH, Lau J. In an empirical evaluation of the funnel plot, researchers could not visually identify publication bias. $J$ Clin Epidemiol 2005; 58: 894-901.

40. Lisi DM. Applying Recent A1C Recommendations in Clinical Practice. US Pharm 2018; 43: $15-22$.

41. American Diabetes Association.Standards of medical care in diabetes - 2020. Diabetes Care 2020; 43: S66-S76,

42. American Diabetes Association.Standards of medical care in diabetes - 2020. Diabetes Care 2020; 43: S89-S97.

Page $10 / 13$ 
43. Williamson DA, Bray GA, Ryan DH. Is $5 \%$ weight loss a satisfactory criterion to define clinically significant weight loss?. Obesity 2015; 23: 2319-2320.

44. Ference BA, Bhatt AL, Catapano AL. Association of genetic variants related to combined exposure to lower low-density lipoproteins and lower systolic blood pressure with lifetime risk of cardiovascular disease. JAMA 2019; 322: 1381-1391.

45. Wing RR, Lang W, Wadden TA, et al. Benefits of modest weight loss in improving cardiovascular risk factors in overweight and obese individuals with type 2 diabetes. Diabetes Care2011; 34: 1481- 1486.

46. Crowley J, Ball L, Han DY, et al. Doctors' attitudes and confidence towards providing nutrition care in practice: Comparison of New Zealand medical students, general practice registrars and general practitioners. J Prim Health Care. 2015 7: 244-250.

47. Meng Y, Bai H, Wang S, Li Z, Wang Q, Chen L. Efficacy of low carbohydrate diet for type 2 diabetes mellitus management: A systematic review and meta-analysis of randomized controlled trials. Diabetes Res Clin Pract. 2017; 131: 124-131.

48. Sandouk Z, Lansang MC. Diabetes with obesity-Is there an ideal diet?. Cleve Clin J Med. 2017; 84: S4-S14.

49. Wang DD, Hu FB. Precision nutrition for prevention and management of type 2 diabetes. Lancet Diabetes Endocrinol. 2018; 6: 416426.

50. Mitchell LJ, Ball LE, Ross LJ, Barnes KA, Williams LT. Effectiveness of Dietetic Consultations in Primary Health Care: A Systematic Review of Randomized Controlled Trials. J Acad Nutr Diet. 2017; 117: 1941-1962.

51. Huang XL, Pan JH, Chen D, Chen J, Chen F, Hu TT. Efficacy of lifestyle interventions in patients with type 2 diabetes: A systematic review and meta-analysis. Eur J Intern Med. 2016; 27: 37-47.

52. Siopis G, Colagiuri S, Allman-Farinelli M. Dietitians' experiences and perspectives regarding access to and delivery of dietetic services for people with type 2 diabetes mellitus. Heliyon. 2020; 6: e03344.

53. Brown JA, Lee P, Ball L. Time and financial outcomes of private practice dietitians providing care under the Australian Medicare program: A longitudinal, exploratory study. Nutr Diet. 2016; 73: 296-302.

54. Vaillancourt H, Légaré F, Lapointe A, Deschênes SM, Desroches S. Assessing patients' involvement in decision making during the nutritional consultation with a dietitian. Health Expect. 2014; 17: 545-554

55. Endevelt R, Gesser-Edelsburg A. A qualitative study of adherence to nutritional treatment: perspectives of patients and dietitians. Patient Prefer Adherence. 2014; 8: 147-154.

56. Hancock RE, Bonner G, Hollingdale R, Madden AM. 'If you listen to me properly, I feel good': a qualitative examination of patient experiences of dietetic consultations. J Hum Nutr Diet. 2012; 25: 275-284.

57. The Australian Government. Department of Health and Ageing. Medicare benefits schedule. Allied Health Services. (2012). Available from

http://www.mbsonline.gov.au/internet/mbsonline/publishing.nsf/Content/276B8A3E93673BECCA257CCF00051C25/\$File/201212Allied.pdf (accessed on September 5, 2020).

58. National Health Service. Buckinghamshire Healthcare. Nutrition and Dietetics - about your appointment and FAQ. Available from https://www.buckshealthcare.nhs.uk/Our\%20clinical\%20services/A\%20to\%20Z\%20of\%20clinical\%20services/Dietetics/dieteticspatient-information.htm (accessed on September 5, 2020).

59. Howatson A, Wall CR, Turner-Benny P. The contribution of dietitians to the primary health care workforce. J Prim Health Care. 2015; 7: 324-332.

60. Lammers M, Kok L. Cost-benefit analysis of dietary treatment. Commissioned by the Dutch Association of Dietitians; 2012. Version 22. SEO Report No. 2012-76A. ISBN 978-90-6733-668-0.

61. Siopis $G$, Jones A, Allman-Farinelli $M$. The dietetic workforce distribution geographic atlas provides insight into the inequitable access for dietetic services for people with type 2 diabetes in Australia. Nutr Diet. 2020; 77: 121-130.

62. Egger M, Zellweger-Zähner T, Schneider M, Junker C, Lengeler C, Antes G. Language bias in randomised controlled trials published in English and German. Lancet 1997; 350: 326-329.

63. Grégoire G, Derderian F, Le Lorier J. Selecting the language of the publications included in a meta-analysis: is there a Tower of Babel bias?. J Clin Epidemiol. 1995; 48: 159-163.

64. Thompson SG, Pocock SJ. Can meta-analyses be trusted? Lancet 1991; 338: 1127-1130.

65. Lau J, loannidis J P, Schmid C H. Summing up evidence: one answer is not always enough. Lancet 1998; 351: 123-127.

\section{Tables}


Due to technical limitations, table 1 and 2 is only available as a download in the Supplemental Files section.

\section{Figures}

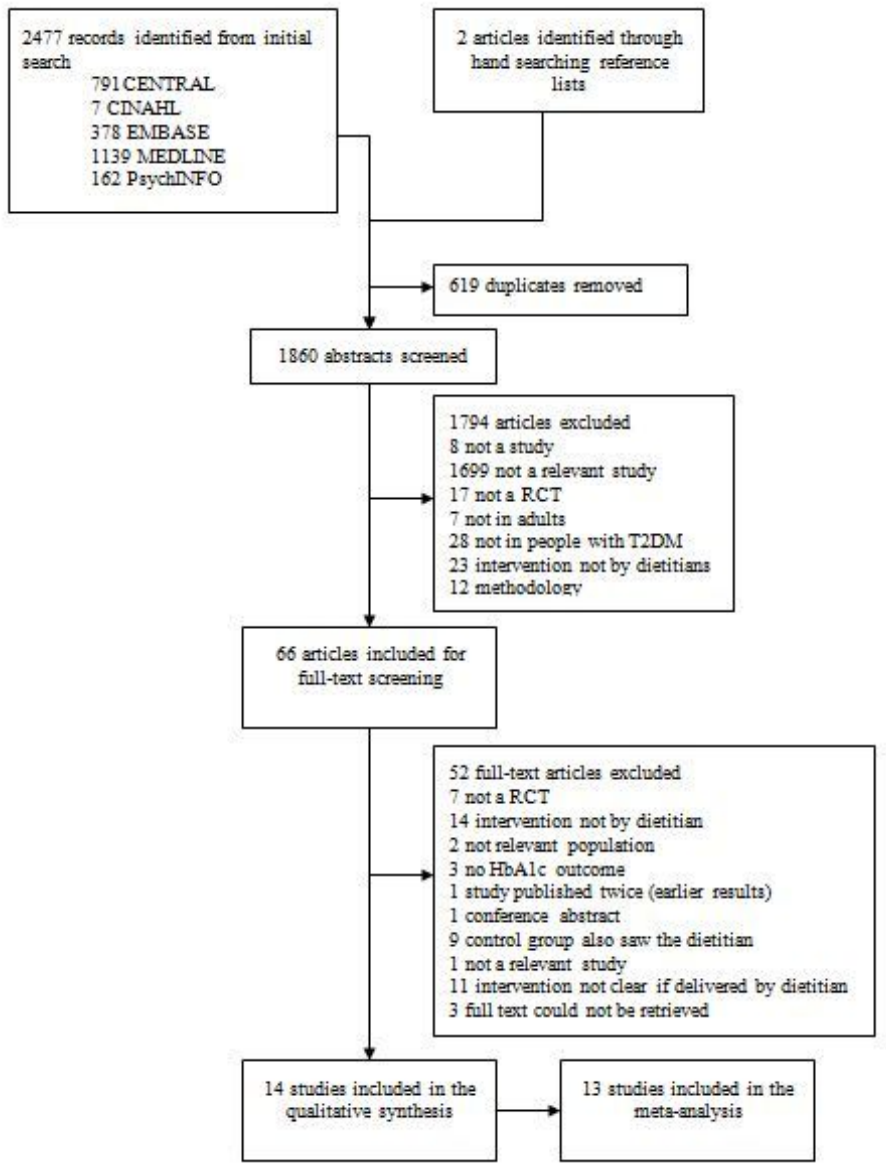

\section{Figure 1}

Study selection RCT=randomized controlled trial. T2DM=type 2 diabetes mellitus. HbA1c=haemoglobin A1c. 


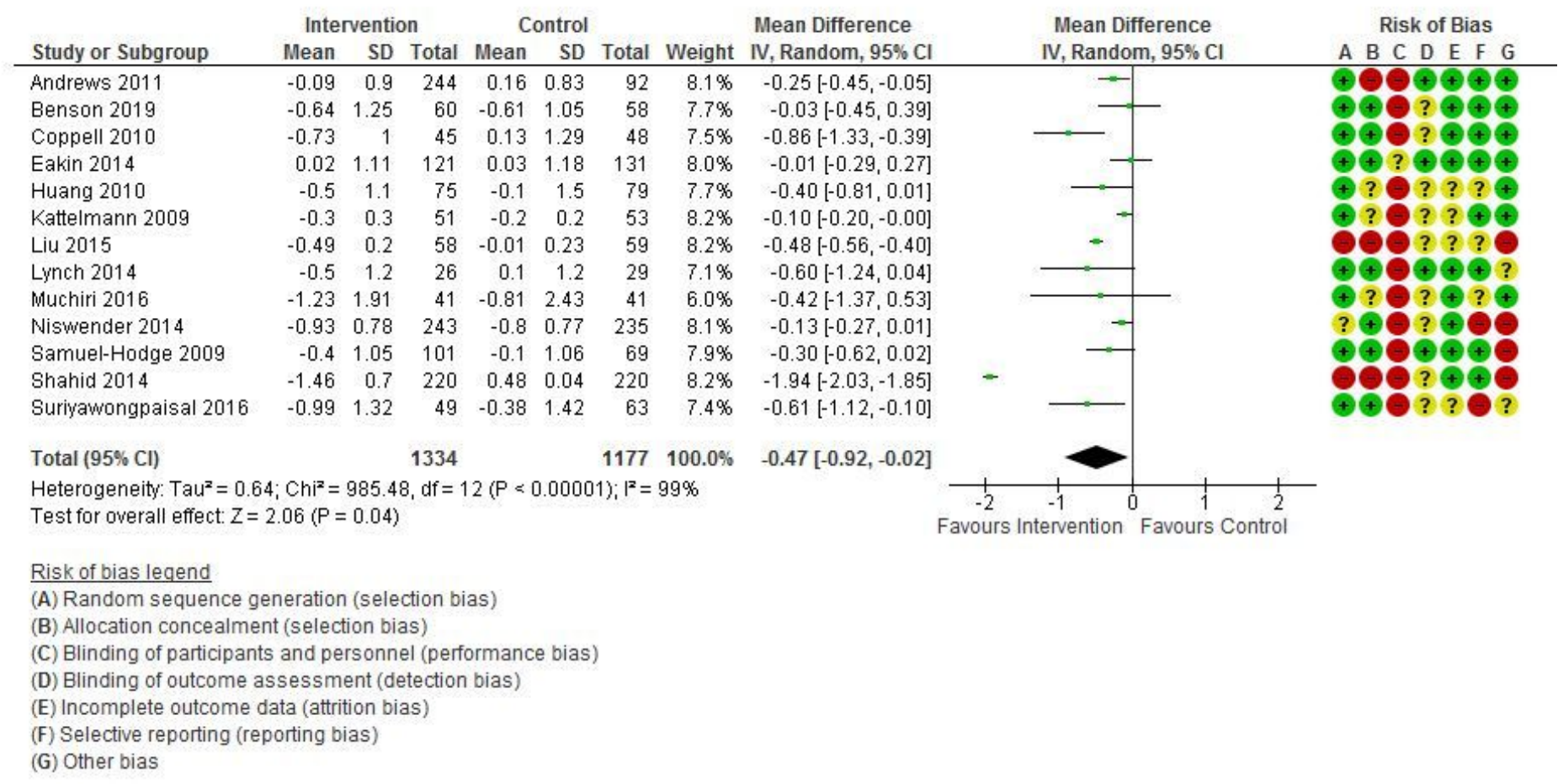

\section{Figure 2}

$\mathrm{HbA1c}$ meta-analysis Forest plot of Mean difference in $\mathrm{HbA} 1 \mathrm{c}$ (expressed as \%) during medical nutrition therapy delivered by accredited dietitians compared with nutrition advice delivered by other healthcare professionals. The size of the green squares indicates the weight of the evidence from each of the studies; studies with confidence interval (horizontal line) crossing zero (vertical line) are inconclusive; powerful studies (those with more participants) have narrower confidence intervals; the black diamond represents the summary effect size, with the width of the diamond indicating the $95 \% \mathrm{Cl}$; a statistically greater significant reduction in $\mathrm{HbA} 1 \mathrm{c}$ is seen in the intervention group, compared to the control group. The data present substantial heterogeneity. Risk-of-bias categories traffic light interpretation is as follows: green (+), low risk; red (-), high risk; no colour, unclear risk (information missing). References listed are Andrews et al. (24), Benson et al. (25), Coppell et al. (26), Eakin et al. (27), Huang et al. (28), Kattelmann et al. (29), Liu et al. (30), Lynch et al. (31), Muchiri et al. (33), Niswender et al. (34), Samuel-Hodges et al. (35), Shahid et al. (36) and Suriyawongpaisal et al. (37). HbA1c=haemoglobin A1c. $\mathrm{IV}=$ inverse variance. $95 \% \mathrm{Cl}=95 \%$ confidence interval.

\section{Supplementary Files}

This is a list of supplementary files associated with this preprint. Click to download.

- Table2SummaryofFindings.docx

- PRISMA2009checklist.doc

- Supplementarymaterial.docx

- Table10verviewofcharacteristicsofincludedstudies.docx 